\title{
Effects of extrinsic and intrinsic perturbations on the electronic structure of graphene: Retaining an effective primitive cell band structure by band unfolding
}

\author{
Paulo V. C. Medeiros, Sven Stafström and Jonas Björk
}

\section{Linköping University Post Print}

\section{Tweet}

N.B.: When citing this work, cite the original article.

Original Publication:

Paulo V. C. Medeiros, Sven Stafström and Jonas Björk, Effects of extrinsic and intrinsic perturbations on the electronic structure of graphene: Retaining an effective primitive cell band structure by band unfolding, 2014, Physical Review B. Condensed Matter and Materials Physics, (89), 4, 041407-1-041407-4.

http://dx.doi.org/10.1103/PhysRevB.89.041407

Copyright: American Physical Society http://www.aps.org/

Postprint available at: Linköping University Electronic Press

http://urn.kb.se/resolve?urn=urn:nbn:se:liu:diva-103755 


\title{
Effects of extrinsic and intrinsic perturbations on the electronic structure of graphene: Retaining an effective primitive cell band structure by band unfolding
}

\author{
Paulo V. C. Medeiros, ${ }^{*}$ Sven Stafström, and Jonas Björk ${ }^{\dagger}$ \\ Department of Physics, Chemistry and Biology, IFM, Linköping University, 58183 Linköping, Sweden
}

(Received 17 October 2013; revised manuscript received 13 December 2013; published 24 January 2014)

\begin{abstract}
We use a band unfolding technique to recover an effective primitive cell picture of the band structure of graphene under the influence of different types of perturbations. This involves intrinsic perturbations, such as structural defects, and external ones, comprising nitrogen substitutions and the inclusion of graphene in adsorbed systems. In such cases, the band unfolding provides a reliable and efficient tool for quantitatively analyzing the effect of doping and defects on the electronic structure of graphene. We envision that this approach will become a standard method in the computational analysis of graphene's electronic structure in related systems.
\end{abstract}

DOI: 10.1103/PhysRevB.89.041407

PACS number(s): 73.22.Pr, 71.20.-b, 73.20.At, 73.20.Hb

Graphene, the two-dimensional carbon allotrope [1], has a unique combination of properties which make it highly interesting for a wide range of applications, such as flexible high performance electronics, spintronics, photodetectors, different types of sensors, etc. [2]. The underlying physical properties, such as electrical and thermal conductivity, mechanical stiffness, strength, and elasticity, and optical properties, have been the subject of numerous studies since the discovery in 2004 $[3,4]$.

In order to use graphene for electronics, however, it is often necessary to restrain its properties. This can be performed, for instance, by patterning, e.g., formation of graphene nanoribbons [5], or by doping [2]. The latter is commonly achieved by introducing nitrogen or boron as substitutional impurities [6]. An alternative route for tailoring graphene's characteristics is to exploit structural defects, which can be introduced in a controlled way under certain conditions [6].

In several practical situations, both doping and structural defects can be seen as perturbing agents modifying the properties of pristine graphene. Assessing how graphene's electronic structure changes under the influence of such perturbations is thus of major interest. In particular, in order for computational investigations of the influence of such external agents on the electronic dispersion to come reliable and efficient, unambiguous evaluations of graphene's band structure under their influence are crucial.

Calculations involving realistic systems are frequently performed using a supercell (SC) approach. Despite the equivalence between the primitive cell (PC) and the SC descriptions of a perfectly periodic material, the latter brings in an inconvenience: The folding of the bands into the smaller SC Brillouin zone (SCBZ) gives rise to complicated band structures, even for relatively small SCs. This is illustrated in Fig. 1 for a calculation using a $4 \times 4$ graphene SC, for which the band structure is already difficult to relate to the one of the PC. Unlike this trivial example, using a PC is not possible in the cases of the perturbed structures discussed above. We show in this Rapid Communication that a band unfolding technique constitutes an extremely efficient tool to simplify the analysis of the results. Although the unfolding is strictly exact only for perfect SCs, it can still provide a meaningful physical representation, in terms of an effective PC band structure (EBS), if the deviations from the perfect case are small $[7,8]$.

Here we introduce the band unfolding technique to recover an effective PC picture of graphene's band structure from calculations using different SCs which include both intrinsic and extrinsic perturbations. We performed the unfolding using our implementation [9], based on the method by Popescu and Zunger [10]. The periodic density functional theory (DFT) calculations were carried out using the VASP code [11-14], with a kinetic energy cutoff of $400 \mathrm{eV}$. Specific computational details for each of the considered systems are given in the Supplemental Material [15]. The theory behind the band unfolding methodology has been described thoroughly elsewhere [7,8,10,16-19], and only a brief summary is presented here.

We first define $\Omega_{\mathrm{PCBZ}}$ and $\Omega_{\mathrm{SCBZ}}$ as the volumes of the primitive cell Brillouin zone (PCBZ) and SCBZ, respectively. For each wave vector $\vec{K}$ of the SCBZ, there are $N_{\text {unfold }} \equiv$ $\Omega_{\mathrm{PCBZ}} / \Omega_{\mathrm{SCBZ}}$ wave vectors $\vec{k}_{i}$ of the PCBZ such that

$$
\vec{k}_{i}=\vec{K}+\vec{G}_{\vec{k}_{i} \leftarrow \vec{K}}, \quad i=1,2,3, \ldots, N_{\text {unfold }},
$$

where the vectors $\vec{G}_{\vec{k}_{i} \leftarrow \vec{K}}$ belong to the SC reciprocal lattice. The wave vector $\vec{K}$ is said to unfold onto $\vec{k}_{i}$ with the unfolding vector $\vec{G}_{\vec{k}_{i} \leftarrow \vec{K}}$. The reverse operation is

$$
\vec{K}=\vec{k}-\vec{G}_{\vec{k} \rightarrow \vec{K}},
$$

where $\vec{k}$ folds into $\vec{K}$ with the folding vector $\vec{G}_{\vec{k} \rightarrow \vec{K}}$. Note that, for a given $\vec{k}$, there is only one $\vec{K}$ that satisfies this relation.

Now consider an eigenstate $\left|\psi_{m \vec{K}}^{\mathrm{SC}}\right\rangle$ of the Hamiltonian in the SC representation. Let $\left\{\overrightarrow{\widetilde{k}}_{i}\right\}$ be the set of wave vectors $\vec{k}_{i}$ in the PCBZ, which relate to $\vec{K}$ through Eq. (1), and correspond to PC eigenstates $\left|\psi_{n \vec{k}_{i}}^{\mathrm{PC}}\right\rangle$ with the same eigenvalue as $\left|\psi_{m \vec{K}}^{\mathrm{SC}}\right\rangle$. The eigenstates $\left|\psi_{m \vec{K}}^{\mathrm{SC}}\right\rangle$ and $\left|\psi_{n \vec{k}_{i}}^{\mathrm{PC}}\right\rangle$ are related as $[10,17,20]$

$$
\left|\psi_{m \vec{K}}^{\mathrm{SC}}\right\rangle=\sum_{\substack{n \\ \vec{k}_{i} \in\left\{\overrightarrow{\vec{k}}_{i}\right\}}} a\left(\vec{k}_{i}, n ; \vec{K}, m\right)\left|\psi_{n \vec{k}_{i}}^{\mathrm{PC}}\right\rangle .
$$

\footnotetext{
*paume@ifm.liu.se

†jonas.bjork@liu.se
} 


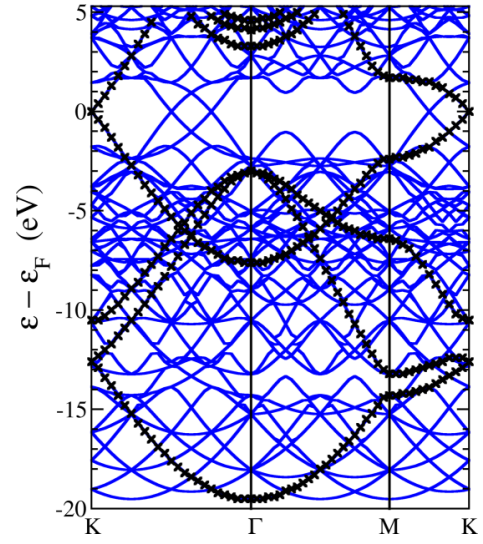

FIG. 1. (Color online) Band structure for a $4 \times 4 \mathrm{SC}$ of graphene, before (blue lines) and after ( $\times$ marks) unfolding, along the highsymmetry directions of graphene's PCBZ.

The probability of $\left|\psi_{m \vec{K}}^{\mathrm{SC}}\right\rangle$ having the same character as a PC Bloch state of wave vector $\vec{k}$ is given by the spectral weight $P_{m \vec{K}}(\vec{k})[10,17]:$

$$
P_{m \vec{K}}(\vec{k}) \equiv \sum_{n}\left|\left\langle\psi_{m \vec{K}}^{\mathrm{SC}} \mid \psi_{n \vec{k}}^{\mathrm{PC}}\right\rangle\right|^{2}=\sum_{\vec{g} \in \mathrm{PCRL}}\left|C_{m \vec{K}}^{\mathrm{SC}}(\vec{g}+\vec{k}-\vec{K})\right|^{2},
$$

where PCRL stands for the PC reciprocal lattice. The second equality in Eq. (4) shows that $P_{m \vec{K}}(\vec{k})$ can be obtained entirely from the coefficients $C_{m \vec{K}}^{\mathrm{SC}}$ of the plane waves that span the eigenstates of the $\mathrm{SC}$, which means that the knowledge of the $\mathrm{PC}$ eigenstates is not required [10]. The spectral function $A(\vec{k} ; \varepsilon)$ is then defined as $[10,19]$

$$
A(\vec{k} ; \varepsilon) \equiv \sum_{m} P_{m \vec{K}}(\vec{k}) \delta\left(\varepsilon-\varepsilon_{m}(\vec{K})\right) .
$$

It is clear that the only pairs $(\vec{k}, \vec{K})$ that need to be included in the sum in Eq. (5) are those in which $\vec{K}$ unfolds onto $\vec{k}$. The last step is then using the spectral function to obtain the EBS. Slightly differently from what is done in Refs. [8,10], we accomplish this by working with the infinitesimal version $d S_{\vec{k}}(\varepsilon)=A(\vec{k} ; \varepsilon) d \varepsilon$ of the cumulative probability function $S_{\vec{k}}(\varepsilon)$. By observing that $d S_{\vec{k}}(\varepsilon)$ represents the number of PC bands, at the PC wave vector $\vec{k}$, crossing the energy interval $(\varepsilon, \varepsilon+d \varepsilon)$, we map the region of interest in the $(\vec{k} ; \varepsilon)$ space onto a $\left(\vec{k}_{i}, \varepsilon_{j}\right)$ grid with energy intervals of size $\delta \varepsilon$, and assign a weight $\delta N\left(\vec{k}_{i} ; \varepsilon_{j}\right)$ to each point, where the quantity

$$
\begin{aligned}
\delta N\left(\vec{k}_{i} ; \varepsilon_{j}\right) & \equiv \int_{\varepsilon_{j}-\delta \varepsilon / 2}^{\varepsilon_{j}+\delta \varepsilon / 2} d S_{\vec{k}_{i}}(\varepsilon) \\
& =\sum_{m} P_{m \vec{K}}\left(\vec{k}_{i}\right) \int_{\varepsilon_{j}-\delta \varepsilon / 2}^{\varepsilon_{j}+\delta \varepsilon / 2} \delta\left(\varepsilon-\varepsilon_{m}(\vec{K})\right) d \varepsilon
\end{aligned}
$$

gives the number of PC bands crossing $\left(\vec{k}_{i} ; \varepsilon_{j}\right)$. The advantage with this approach is that we do not need algorithms for finding the peaks of $A(\vec{k} ; \varepsilon)$, nor the steps of $S_{\vec{k}}(\varepsilon)$. Finally, $\delta N$ is averaged over wave vectors $\vec{k}_{i}$ related by symmetry operations of the PCBZ.
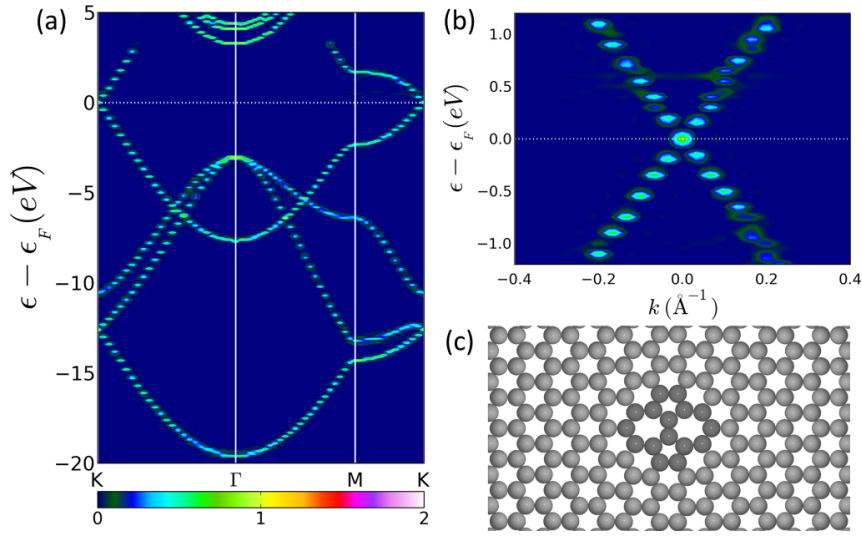

FIG. 2. (Color online) SW defect in graphene: (a) and (b) EBS, and (c) equilibrium geometry. In (b), the $k$ axis is perpendicular to the $K-\Gamma$ direction, and the point $K$ is at $k=0$. The color scale represents $\delta N$ (see main text).

We begin by applying the band unfolding method described above to obtain the EBS for graphene under the influence of intrinsic perturbations. We have simulated a Stone-Wales (SW) defect [21] (Fig. 2), as well as a $V_{2}$ (555-777) double vacancy [6] (Fig. 3). The SW defects are normally formed by exposing graphene to nonequilibrium conditions, but since a barrier of about $5 \mathrm{eV}$ is needed to revert it, it tends to remain stable once the equilibrium conditions are restored [6]. The $V_{2}(555-777)$ defect was chosen since it has shown to be thermodynamically more favorable than other types of double vacancies [6].

Figure 2(a) shows the EBS for the case of the SW defect. The only appreciable difference between the EBS and the actual pristine graphene's PC band structure (PCBS) is the smearing of the PC eigenvalues $\varepsilon_{n}^{\mathrm{PC}}(\vec{k})$ : A set of energy levels $\varepsilon_{n}^{(i)}(\vec{k})$ now appears smeared around band centers $\widetilde{\varepsilon}_{n}(\vec{k}) \approx \varepsilon_{n}^{\mathrm{PC}}(\vec{k})$ with smearing widths $\Delta \varepsilon_{n}(\vec{k})$. The summation of $\delta N$ for these energy levels gives approximately the number of $\mathrm{PC}$ bands crossing $(\vec{k} ; \varepsilon)$ for pristine graphene (1 or 2). As shown in Fig. 2(b), the Dirac cone remains preserved after introducing the defect.
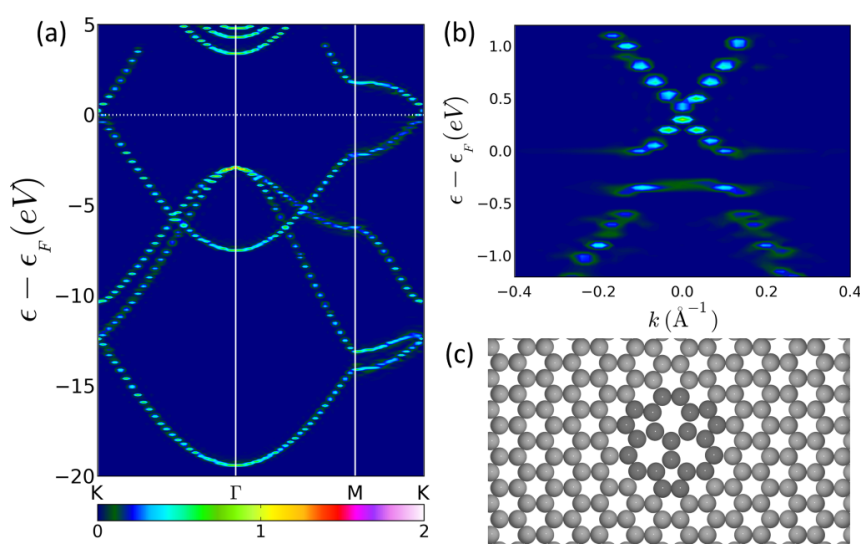

FIG. 3. (Color online) $V_{2}(555-777)$ defect in graphene: (a) and (b) EBS, and (c) equilibrium geometry. In (b), the $k$ axis is perpendicular to the $K-\Gamma$ direction, and the point $K$ is at $k=0$. The color scale represents $\delta N$ (see main text). 
The EBS for the $\mathrm{V}_{2}$ (555-777) defect is shown in Fig. 3(a). Although it appears to retain the overall shape of graphene's PCBS, a closer look into the region shown in Fig. 3(b) reveals the appearance of two flat bands - one around the Fermi energy and another located around $0.36 \mathrm{eV}$ below it. We attribute this effect to the electron localization around the defective region [22] (see the band-decomposed charge densities included in the Supplemental Material [15]). Such redistribution of electronic states is accomplished at the cost of breaking the lower branch of the Dirac cone. The transfer of electrons from graphene's $\pi$ band to the defective region is reflected by an upwards shift of the Dirac point with respect to the Fermi level.

From the calculated $\delta N(\vec{k} ; \varepsilon)$, one can determine both $\widetilde{\varepsilon}_{n}(\vec{k})$ and $\Delta \varepsilon_{n}(\vec{k})$ (see Supplemental Material [15]). We illustrate, for different concentrations of SW and $\mathrm{V}_{2}$ (555-777) defects, that $\Delta \varepsilon_{n}(\vec{k})$ can be used to quantify the smearing of the bands. It is clear that an increased defect density leads to more spread bands, while the positions of the band centers remain relatively constant.

As an example of an extrinsic perturbation, we analyzed the case of a double nitrogen substitution in graphene. The employed SC contains $798 \mathrm{C}$ atoms and one $\mathrm{N}_{2}^{A A}$ dopant, reproducing the experimentally measured dopant concentration of around $5 \times 10^{12} \mathrm{~N}_{2}^{A A}$ defects per $\mathrm{cm}^{2}$ reported in Ref. [23]. A Fermi level shift of $0.28 \mathrm{eV}$ can be readily observed from the EBS [Fig. 4(b)], in close agreement with the experimentally estimated shift of $0.35 \mathrm{eV}$ [23]. Furthermore, some defectinduced flat bands can now be identified, indicating once again electronic localization around the defect. As in the case of the SW defect, smearing out of the energy levels is also observed. Besides the $\mathrm{N}_{2}^{A A}$ defect, we have also studied the case of a single $\mathrm{N}$ dopant with a setup corresponding to the experimental conditions reported in Ref. [24]. We found similar results for this case, also in agreement with the corresponding experimental results (see Supplemental Material [15]). We would like to stress that the simplicity of the analysis and its interpretation is made possible as a result of the band unfolding method.

The effectiveness of the method is further accentuated when we consider the band structure of graphene in adsorption
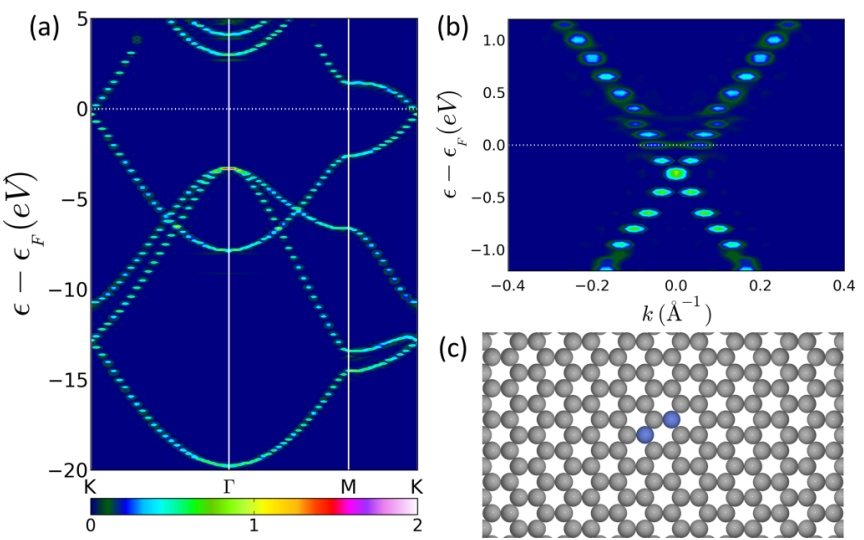

FIG. 4. (Color online) $\mathrm{N}_{2}^{A A}$ substitutional defect in graphene: (a) and (b) EBS, and (c) equilibrium geometry. In (b), the $k$ axis is perpendicular to the $K-\Gamma$ direction, and the point $K$ is at $k=0$. The color scale represents $\delta N$ (see main text).
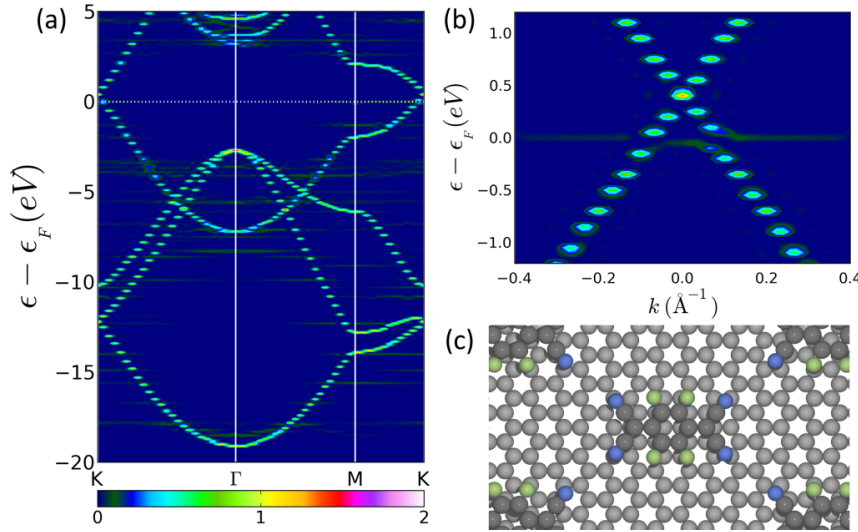

FIG. 5. (Color online) F4-TCNQ@graphene: (a) and (b) EBS, and (c) equilibrium geometry. In (b), the $k$ axis is perpendicular to the $K-\Gamma$ direction, and the point $K$ is at $k=0$. The color scale represents $\delta N$ (see main text).

complexes. As an example, we simulated a 2,3,5,6-tetrafluoro7,7,8,8-tetracyanoquinodimethane (F4-TCNQ) molecule adsorbed on graphene, and, as in Ref. [25], we used a $6 \times 6$ graphene SC. The EBS [Fig. 5(a)] leads to the same quantitative conclusions as those drawn in Ref. [25], regarding both the $p$-type doping of graphene and the presence of a molecule-derived energy level at $E_{F}$. An immediate advantage of our approach for this type of system is that, by providing a unified reference frame for calculations using different-sized $\mathrm{SCs}$, it allows a much more straightforward investigation of the changes in the band structure of graphene as a function of molecular coverage.

Finally, we investigated the adsorption of graphene on a $\mathrm{Cu}(111)$ surface (Fig. 6). Due to the incommensurability of these two lattices, we adjusted the metal's in-plane lattice parameter to match graphene. Such an adjustment could, in principle, introduce artificial effects in the electronic structure of the system. To avoid this problem, we used a relatively large SC, shown in Fig. 6(c), for which the mismatch is only $0.6 \%$. Figure 6 (a) shows the EBS for this system.
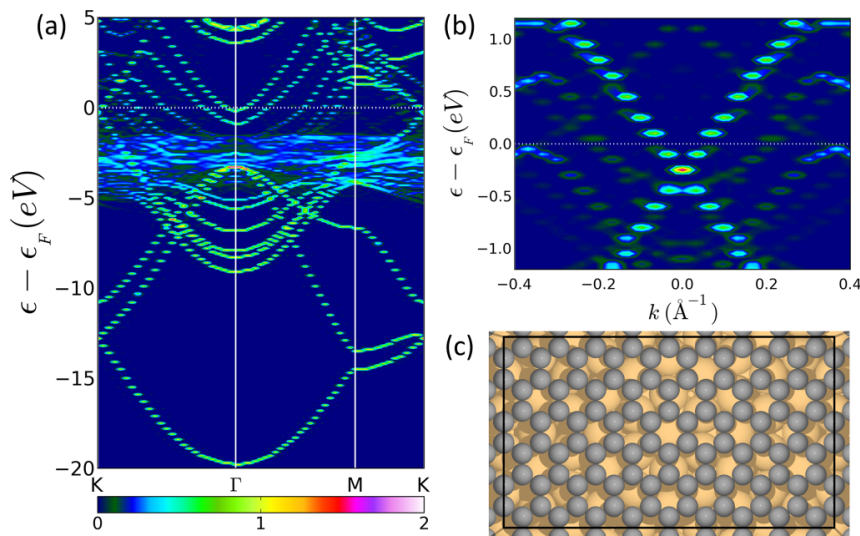

FIG. 6. (Color online) Graphene @ Cu(111): (a) and (b) EBS, and (c) employed SC. In (b), the $k$ axis is perpendicular to the $K-\Gamma$ direction, and the point $K$ is at $k=0$. The color scale represents $\delta N$ (see main text). 
While a direct comparison with graphene's PCBS would be virtually impossible without performing band unfolding, the characteristic PCBS of graphene can be easily identified among the copper bands in the EBS. A simple inspection of the position of graphene's Dirac point in Fig. 6(b) reveals the type and amount of doping of graphene ( $n$ type, with a Fermi level shift of $0.25 \mathrm{eV}$ ).

In conclusion, we have applied a band unfolding technique to analyze the electronic structure of graphene in the presence of intrinsic and extrinsic perturbations. The suitability of such a methodology was demonstrated for cases where an effective PC picture of the band structure of graphene is desired, but the use of a PC is not possible. Notably, this methodology can be employed to study any periodic system. A major advantage of this approach is that it provides a better interface for comparing electronic dispersion relations obtained for systems that, albeit sharing a similar nature, are simulated using different SCs. This includes, for example, obtaining rules on how the density of a particular defect affects the band structure. Furthermore, reporting not only SC-derived band structures but also the EBS obtained from band unfolding establishes a common ground in which theory and experiments can be better faced against each other, as experimental band structure measurements, such as angle-resolved photoemission spectroscopy (ARPES), are often represented along the highsymmetry directions of the graphene PCBZ. We envision that the band unfolding technique will be routinely applied to unambiguously calculate perturbed band structures of graphene, both for comparison with experimental data, as well as for computational screening of novel graphene-based electronic materials.

The authors acknowledge the Swedish Research Council for funding. Computer resources were allocated by the National Supercomputer Centre, Sweden, through SNAC and the MATTER consortium.
[1] K. S. Novoselov, A. K. Geim, S. V. Morozov, D. Jiang, Y. Zhang, S. V. Dubonos, I. V. Grigorieva, and A. A. Firsov, Science 306, 666 (2004).

[2] K. S. Novoselov, V. I. Fal'ko, L. Colombo, P. R. Gellert, M. G. Schwab, and K. Kim, Nature (London) 490, 192 (2012).

[3] A. K. Geim and K. S. Novoselov, Nat. Mater. 6, 183 (2007).

[4] A. K. Geim, Science 324, 1530 (2009).

[5] J. Cai, P. Ruffieux, R. Jaafar, M. Bieri, T. Braun, S. Blankenburg, M. Muoth, A. P. Seitsonen, M. Saleh, X. Feng, K. Müllen, and R. Fasel, Nature (London) 466, 470 (2010).

[6] F. Banhart, J. Kotakoski, and A. V. Krasheninnikov, ACS Nano 5, 26 (2011).

[7] T. B. Boykin and G. Klimeck, Phys. Rev. B 71, 115215 (2005).

[8] T. B. Boykin, N. Kharche, G. Klimeck, and M. Korkusinski, J. Phys.: Condens. Matter 19, 036203 (2007).

[9] BandUP: Band unfolding code for plane-wave based calculations, www.ifm.liu.se/theomod/compphys/band-unfolding.

[10] V. Popescu and A. Zunger, Phys. Rev. B 85, 085201 (2012).

[11] G. Kresse and J. Furthmüller, Phys. Rev. B 54, 11169 (1996).

[12] J. P. Perdew, K. Burke, and M. Ernzerhof, Phys. Rev. Lett. 77, 3865 (1996).

[13] M. Dion, H. Rydberg, E. Schroder, D. C. Langreth, and B. I. Lundqvist, Phys. Rev. Lett. 92, 246401 (2004).

[14] J. Klimeš, D. R. Bowler, and A. Michaelides, J. Phys.: Condens. Matter 22, 022201 (2010).
[15] See Supplemental Material at http://link.aps.org/supplemental/ 10.1103/PhysRevB.89.041407 for more information, computational details, and complementary results.

[16] T. G. Dargam, R. B. Capaz, and B. Koiller, Phys. Rev. B 56, 9625 (1997).

[17] L.-W. Wang, L. Bellaiche, S. H. Wei, and A. Zunger, Phys. Rev. Lett. 80, 4725 (1998).

[18] W. Ku, T. Berlijn, and C.-C. Lee, Phys. Rev. Lett. 104, 216401 (2010).

[19] Y. Zhang and L.-W. Wang, Phys. Rev. B 83, 165208 (2011).

[20] L. Bellaiche, S.-H. Wei, and A. Zunger, Phys. Rev. B 56, 10233 (1997).

[21] A. Stone and D. Wales, Chem. Phys. Lett. 128, 501 (1986).

[22] M. Hjort and S. Stafström, Phys. Rev. B 61, 14089 (2000).

[23] R. Lv, Q. Li, A. R. Botello-Méndez, T. Hayashi, B. Wang, A. Berkdemir, Q. Hao, A. L. Elías, R. Cruz-Silva, H. R. Gutiérrez, Y. A. Kim, H. Muramatsu, J. Zhu, M. Endo, H. Terrones, J.-C. Charlier, M. Pan, and M. Terrones, Sci. Rep. 2, 586 (2012).

[24] L. Zhao, R. He, K. T. Rim, T. Schiros, K. S. Kim, H. Zhou, C. Gutiérrez, S. P. Chockalingam, C. J. Arguello, L. Pálová, D. Nordlund, M. S. Hybertsen, D. R. Reichman, T. F. Heinz, P. Kim, A. Pinczuk, G. W. Flynn, and A. N. Pasupathy, Science 333, 999 (2011).

[25] H. Pinto, R. Jones, J. P. Goss, and P. R. Briddon, J. Phys.: Condens. Matter 21, 402001 (2009). 